\title{
Psicología de la salud en el contexto del conflicto armado en Colombia: un marco de referencia*
}

\author{
Health's Psychology in the Colombian armed conflict context: a framework
}

Recibido: 13 de septiembre de 2015/Aceptado: 3 de agosto de 2016

http://doi.org/10.17081/psico.20.37.2427

\author{
Ara Mercedes Cerquera Córdoba ${ }^{1}$, Arbey Jair Peña Peña ${ }^{2}$ \\ Carol Juliana García Vargas ${ }^{3}$, Diego Andrés Orejuela Castro ${ }^{4}$ \\ Miguel Ángel García Nova ${ }^{5}$ \\ Universidad Pontificia Bolivariana, Colombia
}

Palabras clave:

Conflicto armado, Dolor,

Víctimas, Calidad de vida.

Key words:

Armed Conflict, Pain,

Victims, Quality of life.

\begin{abstract}
Resumen
La población civil es la principal víctima de la crisis humanitaria causada por el conflicto armado que se vive en Colombia desde hace más de 50 años. En este artículo de revisión, se abordarán temas referentes a salud y dolor en este y otros contextos. Metodológicamente, se toman en cuenta datos estadísticos, así como referentes legales, académicos y sociales. Todo esto se lleva a cabo con el propósito de construir un marco de referencia desde el cual se reconozca la pertinencia de la investigación en el campo y se procure que las futuras investigaciones identifiquen la influencia de factores psicosociales en el desarrollo y evolución del estado de 'salud' y 'enfermedad' de las víctimas del conflicto armado en Colombia.
\end{abstract}

\begin{abstract}
The civilian population has been the main victim in the humanitarian crisis caused by the armed conflict in Colombia for more than 50 years. This revision article addresses topics relating to health and pain in this and other contexts. Methodologically, it factors statistical data and legal, academic and social references and issues. This, with the purpose of building a framework from which the pertinence of field research is acknowledged and where future research leads the identification of the influence of psychosocial factors in the development and evolution of "health" and "disease" status of the victims of the armed conflict in Colombia.
\end{abstract}

\section{(c) $(-)$}

Referencia de este artículo (APA):

Cerquera, A., Peña, A., García, C., Orejuela, D. \& García, M. (2017). Psicología de la salud en el contexto del conflicto armado en Colombia: un marco de referencia. Psicogente, 20(37), 181-191. http://doi.org/10.17081/psico.20.37.2427

* Proyecto base: Dolor, calidad de vida y variables asociadas en víctimas del conflicto armado con discapacidad.

1. Doctora en Ciencias Psicológicas, Docente de tiempo completo y Coordinadora de la Especialización en Psicología Clínica, Facultad de Psicología, Universidad Pontificia Bolivariana. Email: ara.cerquera@upb.edu.co http://orcid.org/0000-0002-6773-1495

2. Estudiante, Líder del Semillero de Investigación Calidad de Vida en la Tercera Edad, Facultad de Psicología, Universidad Pontificia Bolivariana. Email: arbey.pena@upb.edu.co http://orcid.org/0000-0001-7668-593X

3. Estudiante, Integrante del Semillero de Investigación Calidad de Vida en la Tercera Edad, Facultad de Psicología, Universidad Pontificia Bolivariana. Email: carol.garcia@upb.edu.co http://orcid.org/0000-0001-6398-2943

4. Estudiante, Facultad de Psicología, Universidad Pontificia Bolivariana. Email: diego.orejuela@upb.edu.co http://orcid.org/0000-0003-4511-950X

5. Estudiante, Facultad de Psicología, Universidad Pontificia Bolivariana. Email: miguel5546@hotmail.com http://orcid.org/0000-0003-3244-6624 


\section{Introducción}

\section{Conflicto armado}

El Centro Nacional de Memoria Histórica adelanta en Colombia un magno proceso de recolección, documentación y gestión comunicativa de memorias y elementos que conforman la historia del conflicto armado; en este marco, para el 2013, se publicó el documento ¡Basta ya! Colombia: Memorias de guerra y dignidad, en el cual se divide el conflicto armado en cinco periodos que abarcan 66 años de desarrollo.

En un primer periodo (1946-1958), se da la fragmentación social y territorial del país. Esta es protagonizada por dos partidos políticos que se disputan el poder, la administración de tierras y recursos, por medio de acciones violentas y atropellos a la libertad de pensamiento en distintas zonas.

Más adelante, en el periodo comprendido entre 1958-1982, denominado 'La Violencia', nacen las primeras guerrillas, las cuales tienen diferentes niveles de organización: al servicio del Gobierno conservador estaban la policía chulavita y Los pájaros (asesinos a sueldo); y en contra de este, las guerrillas liberales y las autodefensas comunistas.

Un tercer momento nos ubica entre los años de 1982 y 1996. En este se expide la Constitución Política del 91 con la que se pretende, entre otros aspectos, contrarrestar la violencia, la corrupción y el enriquecimiento ilícito, a la par que se refuerza el respaldo gubernamental a la ciudadanía, las garantías de participación política y la descentralización de poderes. Este periodo se caracteriza, además, por la expansión de las guerrillas, que imponen normas y estilos de gobierno bajo la premisa del terror. Además, surge el narcotráfico como método de financiamiento tanto de terratenientes como de los grupos al margen de la ley.

Posteriormente, entre 1996 y 2005, se recrudece la lucha del Gobierno contra el narcotráfico, pues, tal como lo expresa Andrés Suárez en una entrevista realizada por el Centro Nacional de Memoria Histórica (2013,10 09), el narcotráfico comienza a encarnar la conciencia de la población colombiana, logrando que los ideales y la dinámica social se tiñan con ideas de progreso mediáticas interesadas por el fin y no por los medios.

Por último, de 2005 a 2012 el proceso del conflicto armado se basa en una gran ofensiva militar del Estado contra las guerrillas y el narcotráfico, en tanto que se producen negociaciones fallidas con los grupos paramilitares que se fortalecieron gracias al narcotráfico. Como resultado de este periodo, el Gobierno comienza a recuperar territorios ocupados durante muchos años por grupos al margen de la ley.

Vale destacar que los actores o líderes del conflicto, además del Gobierno, han sido más de 22 grupos armados y paramilitares, y entre 5 y 10 carteles de la droga y que, según Andrés Suárez, la dinámica del conflicto armado en Colombia se compone de cinco ejes: disputa por la tierra y el conflicto agrario, ausencia de garantías para la participación política, narcotráfico, contexto y presión internacional, y presencia fragmentada del Gobierno en el territorio nacional.

\section{Estadísticas}

En el Reporte general sobre víctimas, de la Unidad para la Atención y Reparación Integral a las Víctimas, hasta el 1 de septiembre de 2015, hay un registro de 
7.620.114 víctimas, lo cual demuestra que en Colombia aproximadamente el $16 \%$ de la población se ha visto afectada (víctima indirecta o directa) por hechos como: Acto terrorista/Atentados/Combates/ Hostigamientos (85.660 personas), Homicidio (265.181 directas y 688.549 indirectas) y Minas antipersonal/Munición sin explotar/Artefacto explosivo (13.305).

\section{Marco legal}

De acuerdo con el artículo 5 de la Ley 975, expedida en el 2005:

Se entiende por víctima la persona que individual o colectivamente haya sufrido daños directos tales como lesiones transitorias o permanentes que ocasionen algún tipo de discapacidad física, psíquica y/o sensorial (visual y/o auditiva), sufrimiento emocional, pérdida financiera o menoscabo de sus derechos fundamentales (p.6)

Además, la Ley 1448 de 2011, en su artículo 3, declara que las víctimas son:

Aquellas personas que individual o colectivamente hayan sufrido un daño por hechos ocurridos a partir del 10 de enero de 1985, como consecuencia de infracciones al Derecho Internacional Humanitario o de violaciones graves y manifiestas a las normas internacionales de Derechos Humanos, ocurridas con ocasión del conflicto armado interno. El cónyuge, compañero o compañera permanente, parejas del mismo sexo y familiar en primer grado de consanguinidad, primero civil de la víctima directa, cuando a esta se le hubiere dado muerte o estuviere desaparecida. A falta de estas, lo serán los que se encuentren en el segundo grado de consanguinidad ascendente. De la misma forma, a las personas que hayan sufrido un daño al intervenir para asistir a la víctima en peligro o para prevenir la victimización (p.9).

\section{Método}

Para la elaboración del presente documento de revisión se utilizaron bases de datos como: SCIELO, REDALYC, EBSCO y SCOPUS; los diferentes artículos de escritura científica, revisiones o lectura crítica consultados hacen parte de revistas indexadas de alto impacto y su fecha de publicación corresponde preferiblemente a los cinco últimos años.

Así pues, el presente documento presenta, en su introducción, algunos elementos que permiten conocer de modo general la dinámica del conflicto armado en Colombia. En esta parte, se acude a las cifras ofrecidas por la Unidad para la Atención y Reparación Integral a las Víctimas, acerca de los hechos y número de personas afectadas; cerrando este primer momento con la concepción de víctima establecida desde el marco legal.

Luego se revisan las principales consecuencias del conflicto armado en Colombia y su significado como fenómeno social; seguido a esto, se tienen en cuenta conceptos claves de la Psicología de la Salud; y, finalmente, bajo una mirada crítica que tiene en cuenta investigaciones y experiencias de intervención en salud, se proponen algunos lineamientos para el abordaje de la salud pública en el contexto del conflicto armado.

\section{Revisión y situación actual}

Para el Estado colombiano, el conflicto armado 
presenta repercusiones a nivel económico, político y social (Cepeda, 2012; Chambers, 2013; Gómez-Isa, 2010; Rojas \& Poveda, 2014; Sandoval \& Martínez, 2010). En este último nivel encontramos los más complejos problemas y brechas de desigualdad.

En el abordaje del problema, los estudios enfatizan en las consecuencias para la urbe, el desplazamiento forzado y la intervención política, judicial y en salud de las partes combatientes (Abello, et al., 2009; Nieto, 2010; Alzate, 2010; Botelho, \& Conde, 2011; Cepeda, 2012; Gómez-Isa, 2010; Jiménez, 2009; León, 2012; Patino \& Patino, 2012; Sandoval \& Martínez, 2010; Villarraga, 2013). Algunos se centran principalmente en los actores y sus acciones (Grupo de Psicología Social Crítica de la Universidad de los Andes [GPSC], 2009) y otros tratan sobre las causas y los móviles de los hechos violentos (García \& Guerrero, 2012; Olave, 2011).

Según Machado y el Programa de las Naciones Unidas para el Desarrollo (2011), en el Informe Nacional de Desarrollo Humano, "el 75,5 \% de los municipios colombianos son rurales, en ellos vive el 31,6\% de la población y representan el 94,4 \% del territorio" (p.32). Por tanto, la población que se ubica en esta zona aporta las principales víctimas y sufren los efectos de una crisis humanitaria que los expone a condiciones violentas en su cotidianidad (Comité Internacional de la Cruz Roja, 2010; Gómez-Isa, 2010; Grupo de Psicología Social Crítica de la Universidad de los Andes, 2009; Machado $\&$ Programa de las Naciones Unidas para el Desarrollo, 2011; Nieto, 2010; Serpa, 2013; Sidel \& Levy, 2009).

Teniendo en cuenta lo anterior, se hace necesario ahondar en la realidad percibida y en la fragmentación de las comunidades del sector rural como consecuencia del conflicto armado.

\section{El conflicto en contexto}

Según Pécaut (2001, citado en Porras-Velásquez, 2011), las situaciones relacionadas con el conflicto armado calan hasta "erosionar las antiguas representaciones sobre las posibilidades de proyectos personales, sobre el presente y el futuro, sobre la estabilidad, situando en su lugar la incertidumbre, la sensación de desarraigo, y el cuestionamiento de las identidades sociales e individuales" (pp.152-153).

Alzate (2010), por su parte, expresa en relación con los estudios publicados hasta la fecha que la violencia y el conflicto interno armado causan un efecto dinamizador (respecto a las motivaciones de movilización y la acción colectiva) y desarticulador (debido a la presión que las amenazas y la violencia directa ejercen en los líderes y activistas sociales).

El Informe General del Centro Nacional de Memoria Histórica (2013) afirma que en las víctimas influyen aspectos de la violencia como: las características de los eventos violentos sufridos, el tipo de victimario, las modalidades de violencia, las particularidades y los perfiles de las víctimas, su edad, género, pertenencia étnica, condición de discapacidad, experiencia organizativa, adscripciones políticas y religiosas, el tipo de apoyo recibido, las respuestas sociales frente a los hechos y a las víctimas, y las acciones u omisiones del Estado, en especial de las fuerzas militares de la policía y de la justicia, pues son los organismos encargados de proteger a la población.

\section{Conceptos claves desde la Psicología de la Salud}

En el marco de la violencia sociopolítica existen investigaciones sobre conceptos que se asocian a variables cognitivo-afectivas indispensables para la compren- 
sión del dolor en esta población partiendo de la idea de bienestar.

El primero de ellos es el fatalismo (Martín-Baró, 1987; Martín-Baró, 1988), concebido como una actitud pasiva con sentimientos de resignación al destino, indefensión y aceptación pasiva de la realidad y sufrimiento causado. El trauma, por su parte, es visto como un conflicto psíquico debido a una experiencia negativa (Hidalgo \&Davidson, 2000; Rubin \& Bloch, 2001) que acarrea la construcción de cogniciones irracionales por la modificación ejercida al sistema cognitivo. Las experiencias traumáticas incrementan, así, la condición de dolor, ocasionando crisis psicológicas, que llevan a una desintegración total del sentido de realidad o a la confusión.

\section{Evaluación de recursos para la intervención}

Diferentes estudios realizados en el mundo (Álvarez, 2012; Giraldo, Toro, Macías, Valencia \& Rodríguez, 2010; Rojas-Rajs \& Soto, 2013) reconocen que las enfermedades causadas por estilos de vida y ambientes insanos, los inadecuados sistemas sanitarios y condiciones socioeconómicas ocasionan un elevado coste económico; por ello, un cambio de enfoque en la salud pública, desde el cual se enfatice en educación sanitaria, en identificación de los determinantes de la salud y en investigación en la calidad de vida, permitirá realizar un compendio de las principales variables personales y sociales que intervienen en el desarrollo de realidades como la salud y la enfermedad (Ávila-Agüero, 2009; Molina, Figueroa $\&$ Uribe, 2013).

De cara a lo anterior, Ávila-Agüero (2009) expone que si se basan las estrategias únicamente en el trabajo del personal médico y sanitario, no se encontrará reso- lución adecuada. En cambio, la acción comunitaria y de diferentes sectores "debe alentar y apoyar el desarrollo de acciones y redes para recoger, transmitir e intercambiar información, para evaluar y desarrollar políticas, estrategias y medidas adecuadas" (p.1). Acorde con esto, Ávila (2013) afirma que la dupla Estado-Empresa Privada juega un papel importante en la atención, acompañamiento y fortalecimiento de sistemas comunitarios y sociales en las zonas afectadas por el conflicto armado. Pero esto último contrasta con la evaluación realizada por Médicos sin Fronteras (MSF, 2013), según la cual, aún no existen programas de atención psicosocial y de salud integral a las víctimas del conflicto armado a la altura de la problemática de salud mental y física y, por tanto, es necesario cuestionar el ejercicio de cada uno de los sectores sociales frente a la realidad y las consecuencias del conflicto. Entonces, si se consideran las conclusiones de las investigaciones, la voz del profesional de la salud se debe hacer oír en el sector económico, administrativo, de justicia, de cultura y ambiente, exigiendo garantías en salud pública; en caso contrario, solo se contará con sistemas inmaduros y excluyentes, que atienden a intereses particulares y que privan, mediatizan y obstaculizan el ejercicio y entrega del saber investigativo y práctico. Acorde con esto, la comisión de determinantes de la salud de la Organización Mundial de la Salud (OMS, 2009) recalca:

La mala salud de los pobres, el gradiente social de salud dentro de los países y las grandes desigualdades sanitarias entre los países están provocadas por una distribución desigual, a nivel mundial y nacional, del poder, los ingresos, los bienes y los servicios, y por las consiguientes injusticias que afectan a las condiciones de vida de la población de forma inmediata y visible (acceso a atención sanitaria, escolarización, educación, condiciones de trabajo y tiempo libre, vivienda, comu- 
nidades, pueblos o ciudades) y a la posibilidad de tener una vida próspera. Esa distribución desigual de experiencias perjudiciales para la salud no es, en ningún caso, un fenómeno 'natural' (p.1).

Tabla 1. Porcentaje de inversión anual en salud del Producto Interno Bruto (PIB)

\begin{tabular}{lccc}
\hline \multicolumn{1}{c}{ País } & 2011 & 2012 & 2013 \\
\hline México & 6,0 & 6,1 & 6,2 \\
Colombia & 6,5 & 6,8 & 6,8 \\
Argentina & 6,3 & 6,8 & 7,3 \\
Brasil & 9,2 & 9,5 & 9,7 \\
Italia & 9,2 & 9,2 & 9,1 \\
Alemania & 11,2 & 11,3 & 11,3 \\
Sudáfrica & 8,6 & 8,9 & 8,9 \\
\hline
\end{tabular}

Fuente: Base de datos de cuentas nacionales de salud de la Organización Mundial de la Salud en Banco Mundial

Continuando con nuestro trabajo, en la Tabla 1, se observa la diferencia entre países en vía de desarrollo y países industrializados en cuanto al respaldo estatal para el acceso equitativo de los servicios por parte de los ciudadanos; lo que finalmente se traduce en justicia social, mayor crecimiento de la actividad económica, del empleo y los ingresos. Colombia, según Serpa (2013), se aproxima a "un empate mutuamente doloroso" (p.1), es decir, un costo altísimo para ambas partes del conflicto, con el agravante de que no hay resolución de este ni preparación para el post-conflicto.

Es urgente atender las secuelas del conflicto (GPSC, 2009), pues el Gobierno debe responder a la deuda que adquirió con la sociedad al abandonar diferentes zonas del país, dejándolas sin garantías de acceso a salud, educación y servicios (Comité Internacional de la Cruz Roja, CICR, 2010; MSF, 2013). De lo contrario, se han de perpetuar políticas y sistemas que priorizan de manera inadecuada el foco de inversión (Serpa, 2013; Sidel \& Levy, 2009), propiciando conflictos y guerras en- tre bandos que han idealizado sus propuestas y no brindan espacios ni garantías para el acuerdo (CICR, 2010).

Debido a lo expuesto hasta aquí, es importante que se ejerza mayor presión al Gobierno en relación a la fiscalización y cobertura de los servicios de salud integral y atención psicosocial prestados, pues estos deben atender la calidad de vida de las víctimas del conflicto y la fragmentación social.

\section{Investigación e intervención desde la Psicología de la Salud}

Tras la revisión acerca de la intervención en salud en distintos contextos, se identifican variables de gran relevancia respecto a bienestar físico y psicológico. Por un lado, Chou et al. (2009) afirman que el dolor tiene impacto negativo en la calidad de vida de los individuos; de igual manera, Serratos-Vázquez, Guevara, García, Roa \& Rivera (2011), al evaluar a 418 pacientes con afectación músculo-esquelética aguda y crónica, encontraron que la presencia de diversas comorbilidades complica el abordaje, la evolución y tratamiento de la enfermedad 'central' por lo que, antes de intervenir, es importante evaluar la percepción de su intensidad.

Además, a partir de los datos encontrados en 86 pacientes con artritis reumatoide de reciente comienzo, Silva et al. (2012) destacan que el afecto negativo representa un estado general de distrés subjetivo como nerviosismo, miedo o disgusto, todo lo cual lleva a estos pacientes a percibir más intensamente el dolor. Sin embargo, Molina et al. (2013) expresan que un adecuado conocimiento de los recursos, herramientas, necesidades, fortalezas y limitaciones, de la mano de acompañamiento y formación emocional, permitirá una ayuda eficaz en la intervención física de los pacientes. 
En cuanto al afrontamiento, Quiceno y Vinaccia (2011) realizaron una investigación orientada a identificar las diferencias de las creencias-prácticas y afrontamiento espiritual-religioso, encontrando que los enfermos crónicos son más espirituales y religiosos. Barragán y Almanza-Rodríguez (2013), por su parte, valoraron las estrategias de afrontamiento del dolor crónico y destacaron que las estrategias de afrontamiento del dolor como esperanza, autoinstrucciones, distracción cognitiva, catastrofización, y fe y plegarias sirven como material complementario para el cuidado de las personas. Por tanto, se deben integrar aspectos físicos o fisiológicos, psicológicos y emocionales en pro de la calidad de vida.

La propuesta y reto se traduce en lo expresado por Molina et al. (2013): "Un equipo de profesionales preparados para atender al ser humano en todas sus dimensiones, podrá impactar mejor la calidad de vida de los pacientes hospitalizados; lo cual a su vez influye en su proceso de recuperación” (p.61). También se debe tomar en cuenta que si la Psicología de la Salud busca trabajar en pro de la identificación de factores psicológicos implicados en el desarrollo, mantenimiento y recuperación de las diversas patologías catalogadas dentro del dolor (Moix \& Casado, 2011), es fundamental que amplie su visión acerca de las concepciones de dolor y aplicar técnicas interdisciplinares, que enriquezcan los procedimientos terapéuticos y mejoren la calidad de vida del paciente.

En tal sentido, Riveros, Castro y Lara-Tapia (2009) determinaron que el área cognitiva/personalidad, social y familiar son partícipes directos del desarrollo de la enfermedad; y algo similar ocurre con la dimensión cognitivo-afectiva, pues es una de las principales herramientas para el afrontamiento de los problemas en relación con el medio (Blair \& Cardona, 2008; Botelho \& Conde, 2011). En cuanto a la dimensión afectivo-emocional, según Silva et al. (2012), la mejor estructuración positiva de los afectos, afecta en forma proporcional la evolución del estado psicológico del paciente ante la enfermedad.

Continuando con lo descrito, en el marco del conflicto armado colombiano, es importante que la Psicología de la Salud identifique relaciones entre salud física y mental. Jiménez (2009) y Patino y Patino (2012) afirman al respecto que el estrés postraumático es uno de los trastornos más frecuentes en excombatientes; en tanto que para Mercado, Molina De Jesús, Hernández \& Méndez (2014), las "víctimas de asalto y violencia desarrollan una expectativa más negativa acerca de su entorno y de las relaciones con otras personas y pueden desarrollar síntomas conductuales de PTSD más claramente identificables que aquellos que fueron víctimas de abuso o maltrato en la niñez" (p.11).

De igual modo, en relación con las personas desplazadas, Abello et al. (2009) señalan que la movilización a lugares desconocidos y sus historias de muerte, tortura y amenaza desintegran el sistema de creencias y generan mayor dificultad para establecer relaciones; disminuyen, además, el crecimiento personal, el interés por desarrollar potencialidades, las convicciones y las expectativas frente a la generación de oportunidades.

Por todo lo anterior, es pertinente acoger el mandato de la Ley 1448 de 2011 en cuanto a la intervención con un enfoque diferencial, pues, cada persona es afectada en distintos niveles y la evaluación de los daños en el orden personal, familiar, comunitario y social dictaminará el modo de actuación desde la Psicología de la Salud. 


\section{Conclusiones}

Para concluir, se destaca la importancia de implementar programas de acompañamiento reflexivo e interventivo, ya que, según lo recalcado por Estrada, Ripoll y Rodríguez (2010), las estrategias conversacionales y los encuentros propios de estos programas logran que los participantes presenten y configuren colaborativamente las necesidades, oportunidades y recursos psico-socio-jurídicos de los que se disponen y animan así los procesos de acompañamiento y reparación.

Desde otra perspectiva, Londoño, Sicachá y González (2011) advierten que las mismas relaciones interpersonales, el rol de las redes de apoyo social y personal, familia, amigos y pares, son determinantes en el proceso de protección y recuperación luego de las experiencias traumáticas.

Por lo tanto, brindar espacios a la narrativa y al análisis de la construcción social en torno a los hechos puede ser una gran herramienta a la hora de resignificar las experiencias, transformar la perspectiva de lo vivido e implementar nuevas estrategias de afrontamiento personal, familiar y comunitario, siendo evidente que tal enfoque de intervención debe ceñirse a un seguimiento y evaluación.

Numerosos estudios exhortan, además, a realizar un análisis de la problemática en el orden público, social y cultural, desde el cual se identifiquen factores de riesgo para la ejecución de programas de apoyo y acompañamiento a diferentes tipos de pacientes. Mediante el trabajo interdisciplinar se adoptarán pautas de responsabilidad y compromiso a nivel político y administrativo, siendo necesario además el acompañamiento social y familiar (Molina et al., 2013) para la evolución positiva de los pacientes. Siendo el dolor un foco de urgente y necesaria observación, es fundamental apostarle a la interdisciplinariedad, pues el multiverso permite tener en cuenta todo lo que influye en el cuidado y mejora de la calidad de vida de la persona.

\section{Referencias}

Abello, R., Amaris, M., Blanco, A., Madariaga, C., Manrique, K., Martínez, M., Turizo, Y. \& Díaz, D. (2009). Bienestar y trauma en personas adultas desplazadas por la violencia política. Revista Universitas Psychologica, 8(2), 455-470.

Álvarez, L. (2012). Los estilos de vida en salud: del individuo al contexto. Revista Facultad Nacional de Salud Pública, 30(1), 95-101.

Alzate, M. (2010). Interpretaciones frente a la violencia y el conflicto armado en Colombia. Estudios Sociales, 18(36), 34-55.

Ávila, C. (2013). Responsabilidad social en proyectos de intervención a territorios afectados por el conflicto armado. Universidad Nueva Granada [Revista electrónica]. 1-17. Recuperado de http://repository. unimilitar.edu.co/bitstream/10654/10976/1/ Ensayo\%20Gesti\%C3\%B3n\%20de\%20Desarro1lo\%20Administrativo.pdf

Ávila-Agüero, M. L. (2009). Hacia una nueva Salud Pública: determinantes de la Salud. Acta Médica Costarricense, 51(2), 71-73.

Barragán, J. A. \& Almanza-Rodríguez, G. (2013). Valoración de estrategias de afrontamiento, a nivel ambulatorio, del dolor crónico en personas con cáncer. Aquichan, 13(3), 322-335. 
Blair, L. P. \& Cardona, M. Á. (2008). Pacientes con cáncer terminal, una mirada fenomenológica existencial. International Journal of Psychological Research, $1(2), 13-20$.

Botelho, S. \& Conde, C. (2011). Memoria emocional y trastorno por estrés postraumático en el contexto del desplazamiento en Colombia. Revista Colombiana de Psiquiatría, 40(3), 457-469.

Centro de Memoria Histórica (2013). Los orígenes, las dinámicas y el crecimiento del conflicto armado. En iBasta ya! Colombia: Memorias de guerra y dignidad. Bogotá: Imprenta Nacional.

Centro Nacional de Memoria Histórica (2013,10,09). Cinco ejes para entender el conflicto armado colombiano. [Archivo de Video]. Recuperado de: https:// www.youtube.com/watch?v=um6GJiOtn64

Cepeda, E. (2012). Corte constitucional y conflicto armado; control de políticas sociales en el marco del conflicto en Colombia. Revista Facultad de Derecho y Ciencias Políticas, 42(117), 425-453.

Chambers, P. (2013). "Guerra civil continuada por otros medios": dimensiones normativas e ideológicas del conocimiento científico-social e histórico en torno al conflicto armado colombiano. Estudios políticos, 42, 37-60.

Chou, R., Loeser, J., Owens, D., Rosenquist, M., Atlas, E., Baisden, J., Carragee, E., Grabois, M., Murphy, D., Resnick, D., Stanos, S., Shaffer, W. \& Wall, E. (2009). Interventional therapies, surgery, and interdisciplinary rehabilitation for low back pain.Spine, 34(10), 1066-1077.
Comité Internacional de la Cruz Roja (2010). Informe, Colombia: consecuencias humanitarias del conflicto armado en Colombia. Recuperado de: http://www.icrc.org/spa/resources/documents/ report/colombia-report-intro-220410.htm

Estrada, A., Ripoll, K. \& Rodríguez, D. (2010). Intervención psicosocial con fines de reparación con víctimas y sus familias afectadas por el conflicto armado interno en Colombia: equipos psicosociales en contextos jurídicos. Revista de Estudios Sociales, 36, 103-112.

García, B. \& Guerrero, B. (2012). Violencia, crisis del capitalismo global y juventud. Revista Colombiana de Educación, 62, 191-219.

Giraldo, A., Toro, M., Macías, A., Valencia, C. \& Rodríguez, S. (2010). La promoción de la salud como estrategia para el fomento de estilos de vida saludables. Revista hacia la Promoción de la Salud, 15(1), 128-143.

Gómez-Isa, F. (2010). La restitución de la tierra y la prevención del desplazamiento forzado en Colombia. Revista Estudios Socio-Jurídicos, 12(2), 11-58.

Grupo de Psicología Social Crítica de la Universidad de los Andes (2009). Del dolor a la propuesta. Voces del panel de víctimas. Revista de Estudios Sociales, 63, 114-125.

Hidalgo, R. \& Davidson, J. (2000). Posttraumatic stress disorder: epidemiology and health-related considerations. Journal of Clinical Psychiatry, 61(7), 5-13.

Jiménez, W. (2009). Salud mental en el postconflicto colombiano. Revista Criminalidad, 51(1), 179-192. 
Recuperado de http://oasportal.policia.gov.co/ imagenes_ponal/dijin/revista_criminalidad/ vol51_1/08salud.pdf

León, A. (2012). Crónica de una vergüenza: el papel de las autoridades civiles y militares durante la llegada de los paramilitares de las AUC al Valle del Cauca (julio-agosto de 1999). Sociedad y Economía, 23, 125-140.

Ley No. 1448 (10 de junio de 2011). Ley de Víctimas y Restitución de tierras. República de Colombia. Recuperado de: http://www.minsalud.gov.co/ Normatividad/Ley\%201448\%20de\%202011.pdf

Ley No. 975 (25 de julio de 2005). Diario Oficial No. 45.980 de la Fiscalía General de la Nación. República de Colombia. Recuperado de: http://www. fiscalia.gov.co:8080/Documentos/LEY_975_ concordada.pdf

Londoño, N., Sicachá, M. \& González, J. (2011). Posibles manifestaciones del trastorno por estrés postraumático en adultos desplazados por el conflicto armado del asentamiento "Acacias Bajo" en Armenia - Quindío. Sinapsis, 3(3), 101-107. Recuperado de: http://www.eam.edu.co/ojs-2.4.2/index.php/prueba/article/view/40/22

Machado, A. \& Programa de las Naciones Unidas para el Desarrollo (2011). Informe de desarrollo humano 2011 - Pnud Colombia rural - razones para la esperanza. Recuperado de: http://escuelapnud.org/ biblioteca/pmb/opac_css/index.php?lvl=notice_ display\&id=617\& seule=1.

Martín-Baró, I. (1987). El latino indolente. Carácter ideológico del fatalismo latinoamericano. En M.
Montero (Coord.), Psicología Politica Latinoamericana (pp.135-162). Caracas: Panapo.

Martín-Baró, I. (1988). La violencia política y la guerra como causas del trauma psicosocial en El Salvador. Revista de Psicología de El Salvador, 7(28), 123. 141.

Médicos Sin Fronteras (2013). Informe. Las heridas menos visibles: salud mental, violencia y conflicto armado en el sur de Colombia.

Mercado D., V., Molina De Jesús, V., Hernández P., L. \& Méndez F., H. (2014). Factores biomédicos y psicológicos para trauma en un grupo de veteranos puertorriqueños. Revista Puertorriqueña de Psicología, 25(1), 80-95. Recuperado de: http:// reps.asppr.net/ojs/index.php/reps/article/ view/232/234

Moix, J. \& Casado, M. J. (2011). Terapias psicológicas para el tratamiento del dolor crónico. Clínica y Salud, 22(1), 41-50.

Molina, J. M., Figueroa, J. \& Uribe, A. F. (2013). El dolor y su impacto en la calidad de vida y estado anímico de pacientes hospitalizados. Universitas Psychologica, 12(1), 55-62.

Nieto, P. (2010). Relatos autobiográficos de víctimas del conflicto armado. Estudios Sociales, 36, 76-8.

Olave, G. (2011). Los marcos del conflicto: Aproximación al conflicto armado colombiano desde el concepto de marco. Discurso E⿱ Sociedad, 5(3), 514 546. Recuperado de: http://www.dissoc.org/ediciones/v05n03/DS5\%283\%29Olave.pdf 
Organización Mundial de la Salud [OMS] (2009). Subsanar las desigualdades en una generación: Alcanzar la equidad sanitaria actuando sobre los determinantes sociales de la salud: Informe final de la Comisión sobre Determinantes Sociales de la Salud. Recuperado de: http://whqlibdoc.who. int/publications/2009/9789243563701_spa. pdf?ua $=1$

Patino, R. \& Patino, C. (2012). Configuración de la identidad de desertores de la guerrilla colombiana. Psicologia $\mathcal{E}$ Sociedade, 24(3), 517-526.

Porras-Velásquez, N. (2011). Lo ideológico en la psicología social y en la guerra en Colombia. Revista de Psicología GEPU, 2(2), 138-157.

Quinceno, J. \&Vinaccia, S. (2011). Creencias-prácticas y afrontamiento espiritual-religioso y características sociodemográficas en enfermos crónicos. Psychologia: Avances de la Disciplina, 5(1), 25-36.

Reporte General sobre Víctimas (mayo 3 de 2014). Unidad para la Atención y Reparación Integral a las Víctimas. Recuperado de: http://www.unidadvictimas.gov.co

Riveros, A., Castro, C. \& Lara-Tapia, H. (2009). Características de la calidad de vida en enfermos crónicos y agudos. Revista Latinoamericana de Psicología, 41(2), 291-304.

Rojas, A. \& Poveda, A. (2014). Conflicto armado, despojo de tierras y actividad ganadera: Indagando entre el testimonio no oficial y las cifras estatales en el departamento del Meta-Colombia. Revista Iberoamericana de Estudios de Desarrollo- Iberoamerican Journal of Development Studies, 3(1), 32-57.
Rojas-Rajs, S. \& Soto, E. (2013). Comunicación para la salud y estilos de vida saludables: aportes para la reflexión desde la salud colectiva. Interface, 17(46), 587-599.

Rubin, B. \& Bloch, E. (2001). Intervención en crisis y respuesta al trauma. Bilbao: Desclée de Brouwer.

Sandoval, L. \& Martínez, D. (2010). Presencia de conflicto armado interno y su efecto en la inversión extranjera directa: Tendencia mundial perspectivas para Colombia (2001-2007). Revista Facultad Ciencias Económicas, 18(1), 133-150.

Serpa, C. (2013, 05, 15). Conflicto armado y post-conflicto. El Heraldo. Disponible en: http://www. elheraldo.co/blogs/politica/conflicto-armado-ypost-conflicto- 110408

Serratos, M., Guevara, U., García, G., Roa, L. \& Rivera, G. (2011). Frecuencia del dolor neuropático en pacientes con lesiones músculo-esqueléticas. Revista Mexicana de Anestesiología, 34(4), 264-269.

Sidel, V. \& Levy, B. (2009). Las consecuencias en salud de la desviación de recursos hacia la guerra y su preparación. Medicina Social, 4(3), 154-157.

Silva, K., Ortiz, A., Patiño, E., Aguilera, C., Velasco, T., García de Vicuña, R. \& González, I. (2012). Influencia de la estructura de los afectos en la evaluación de la artritis reumatoide mediante la escala visual analógica de dolor, el HAQ y el DAS28. Reumatología Clínica, 8(6), 328-333.

Villarraga, A. (2013). Experiencias históricas recientes de reintegración de excombatientes en Colombia. Colombia Internacional, 77, 107-140. 